\title{
Commentary \\ One man's risk factor is another man's outcome: Difference in risk factor profiles for chronic postsurgical pain maintenance vs transition
}

Chronic postsurgical pain develops in an alarming proportion of patients [2,5]. Although we now know more than we ever did about chronic postsurgical pain, we are still in the Dark Ages when it comes to predicting who will go onto develop it and who will recover uneventfully. We know little about the risk and protective factors leading to chronicity. The article by Seebach et al. [9] in this issue of PAIN is noteworthy for its prospective, longitudinal approach to studying the psychosocial recovery trajectory in patients after spine surgery. The authors' main objective was to evaluate the extent to which preoperative and postoperative positive and negative affect predicted several outcomes measured at 6 weeks and 3 months after discharge. They conducted separate regression analyses to evaluate whether positive affect (a putative protective factor) and negative affect and depression scores (two putative risk factors) predicted four related, but different, outcome variables at different times after surgery; namely, pain intensity, pain interference, disability and functional status (after controlling for relevant covariates). The results showed that none of the three putative protective or risk factors predicted any of the four outcome variables 6 weeks and 3 months after surgery. In contrast, 6 -week postoperative positive affect predicted 3-month functional status and 6week negative affect predicted 3-month disability. Moreover, 6week depression scores predicted 3-month pain intensity, pain interference and pain-related disability but not functional status.

The article by Seebach et al. [9] raises three related questions that future research must address if we are to make progress in identifying the causal risk and protective factors involved in the transition from acute to chronic post-surgical pain.

\section{What are the relevant risk and protective factors for a given outcome measure and how are these best determined?}

Predictor variables in one study are often used as outcome measures in other studies and vice versa, reflecting the complexity of the problem and the variety in hypothesized mechanisms. The decision to use a variable as an outcome measure or a predictor should be determined a priori. The challenge is to develop sufficiently broad models of the (biological, psychological, social, cultural, spiritual, etc.) factors believed to be associated with chronic postsurgical outcomes. This requires large numbers of patients. Until recently, the choice of outcome variable has been largely discipline-specific and rather narrow. For example, the more biomedical specialties (e.g., anesthesiology) have tended to use chronic pain intensity or incidence as the main outcome variable whereas the psychological literature has focused more broadly on the impact that the pain has on the person, as measured by pain disability or pain interference. According to this view, pain intensity is a predictor. More recent studies, including that by Seebach et al. [9], have further broadened the outcomes to include the patient's functional status. These advances are due in large part to recommendations [1] to assess core domains of functioning in clinical studies (IMMPACT) although the issue of the most appropriate measure with a given domain remains to be determined. Nevertheless, selecting clinically relevant domains of functioning ultimately will help to identify the causal risk and protective factors that determine whether or not an individual will go on to develop chronic postsurgical pain, related psychosocial dysfunction, and functional impairment.

Do the risk and protective factors involved in the transition to chronicity differ from those involved in the maintenance of already established chronic pain?

The pattern of findings reported by Seebach et al. [9], described above, suggests that the risk and protective factors involved in the transition of acute pain to chronic pain (i.e., baseline to 6 weeks and 3 months postop) differ from the factors involved in maintaining the pain once it has become established (i.e., 6 weeks to 3 months postop). This pattern of results is very similar to what Katz et al. [2] reported in their prospective, longitudinal study of patients after lateral thoracotomy (Fig. 1). Perioperative factors (pre-operative pain disability and emotional numbing and acute postoperative pain intensity) failed to predict pain disability at 6 (i.e., the transition to chronicity) or 12 months after surgery, but the very same factors measured 6 months later were highly significant predictors of (the maintenance of) 12 month pain disability. In spite of the different predictors and outcome variables, the results of both studies suggest that the risk factors that predict the maintenance of established chronic postsurgical pain disability or pain intensity, pain interference, and functional status are different from those that predict the initial transition from acute pain to chronic pain. These data highlight the importance of assessing outcomes at multiple time points after surgery [6] and show that the transition to chronic postsurgical pain and related psychosocial dysfunction is a dynamic process that evolves over time. Assessing outcomes at a single follow-up after surgery will not permit an examination of whether the factors involved in the transition to chronicity differ from those involved in the maintenance of already established chronic pain. 


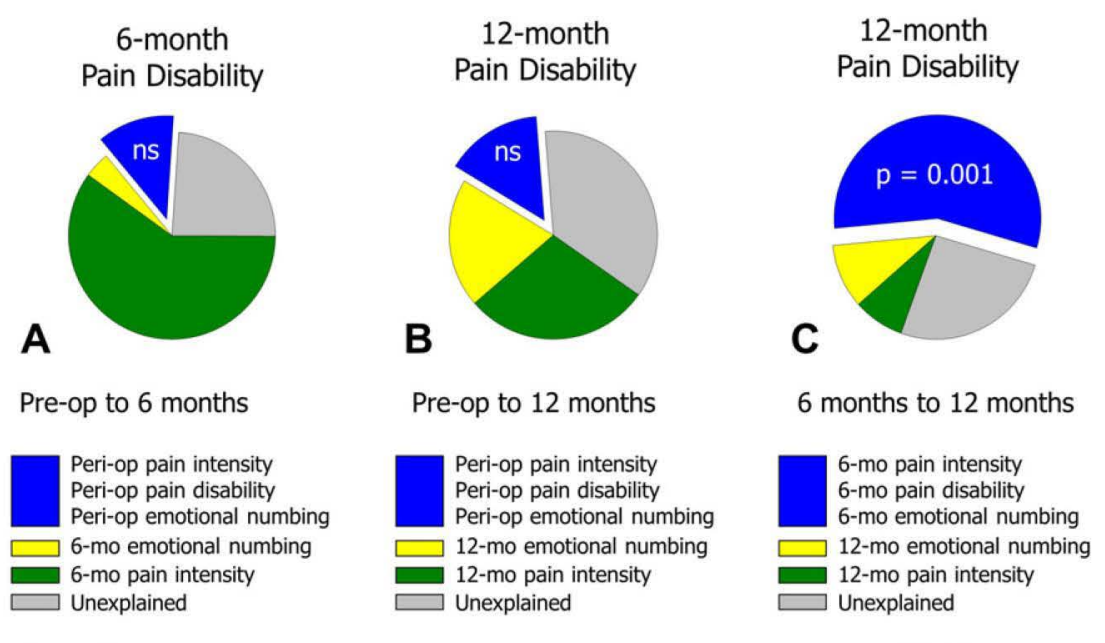

Transition

Maintenance

Fig. 1. Schematic illustration of the change in the proportion of variance in pain disability (outcome) after lateral thoracotomy explained by the very same predictor variables obtained at different points in time. Perioperative factors (pain intensity, pain disability and emotional numbing scores) did not significantly predict pain disability at 6 months (A) or 12 months (B) but the very same factors measured 6 months later were highly significant predictors of pain disability 12 months after surgery (C). These results suggest that the risk factors for maintaining chronic postsurgical pain disability differ from those that are involved in the transition from acute pain to chronic pain disability (Reproduced with permission from Joel Katz, PhD).

\section{What explains the relationship between perioperative pain (preoperative pain and acute postoperative pain) and chronic post-surgical pain?}

The results of the studies by Seebach et al. [9] and Katz et al. [4] are odds with much of the literature on the transition to pain chronicity after surgery in that neither found any preoperative factor to predict later outcomes. This discrepancy is difficult to explain, but one possibility is that the patients who had pain before surgery and/or who were disabled by it were not the ones who went on to develop chronic postsurgical pain and related psychosocial dysfunction. Establishing the nature of the relationship between preoperative pain and chronic postsurgical pain has been at the forefront of clinical and basic research [4,5]. It is also at the heart of Macrae's [7,8] definition of chronic postsurgical pain, the last two points of which are: (a) rule out other causes for the pain, including (b) the possibility that the pain is continuing from a pre-existing problem. Few studies heed this advice and failure to rule out point (b) may well explain the well-established relationship between pre-operative pain and chronic postsurgical pain: If the pain is continuing from a pre-existing problem then one would expect a strong relationship between pain before and after surgery. If not, then pain-related factors in the postoperative period may be more strongly associated with the maintenance of chronic postsurgical pain. Establishing the conditions under which preoperative pain is a causal risk factor for the development of chronic postsurgical pain is central to developing treatments for preventing and managing this costly and distressing problem [3].

\section{Conflict of interest statement}

The author has no conflicts of interests to declare.

\section{Acknowledgment}

J.K. is supported by a Canadian Institutes of Health Research Canada Research Chair in Health Psychology at York University.

\section{References}

[1] Dworkin RH, Turk DC, Farrar JT, Haythornthwaite JA, Jensen MP, Katz NP, Kerns RD, Stucki G, Allen RR, Bellamy N, Carr DB, Chandler J, Cowan P, Dionne R, Galer BS, Hertz S, Jadad AR, Kramer LD, Manning DC, Martin S, McCormick CG, McDermott MP, McGrath P, Quessy S, Rappaport BA, Robbins W, Robinson JP, Rothman M, Royal MA, Simon L, Stauffer JW, Stein W, Tollett J, Wernicke J, Witter J. Core outcome measures for chronic pain clinical trials: IMMPACT recommendations. Pain 2005;113:9-19.

[2] Katz J, Asmundson GJ, McRae K, Halket E. Emotional numbing and pain intensity predict the development of pain disability up to one year after lateral thoracotomy. Eur J Pain 2009;13:870-8.

[3] Katz J, Clarke H, Seltzer Z. Review article: preventive analgesia: quo vadimus? Anesth Analg 2011;113:1242-53.

[4] Katz J, Seltzer Z. Transition from acute to chronic postsurgical pain: risk factors and protective factors. Expert Rev Neurother 2009;9:723-44.

[5] Kehlet H, Jensen TS, Woolf $\mathrm{CJ}$. Persistent postsurgical pain: risk factors and prevention. Lancet 2006:367:1618-25.

[6] Kehlet H, Rathmell JP. Persistent postsurgical pain: the path forward through better design of clinical studies. Anesthesiology 2010;112:514-5.

[7] Macrae WA. Chronic post-surgical pain: 10 years on. $\mathrm{Br} J$ Anaesth 2008:101:77-86.

[8] Macrae WA, Davies HTO. Chronic postsurgical pain. In: Crombie IK, Linton S Croft P, Von Knorff M, LeResche L, editors. Epidemiology of pain. Seattle: IASP Press; 1999. p. 125-42.

[9] Seebach CL, Kirkhart M, Lating JM, Wegener ST, Song Y, Riley III LH, Archer KR. Examining the role of positive and negative affect in recovery from spine surgery. Pain 2012;153:518-25.

Joel Katz

Department of Psychology, York University, Canada Department of Anesthesia and Pain Management, Toronto General Hospital, Canada Mount Sinai Hospital, Department of Anesthesia, University of Toronto, Toronto, ON, Canada * Address: Department of Psychology, York University, 4700 Keele St., BSB 232, Toronto, ON, Canada M3J 1 P3. Tel: $+14167312100 \times 40557$ E-mail address: jkatz@yorku.ca 